\title{
La dependencia emocional como factor de riesgo en la violencia familiar, un problema de salud pública
}

\section{Emotional dependence as a risk factor in family violence, a public health problem}

\author{
Hilda Rosario Beraún Vásquez ${ }^{\text {1,a }}$, Elena Pilar Poma Rojas ${ }^{1, b}$
}

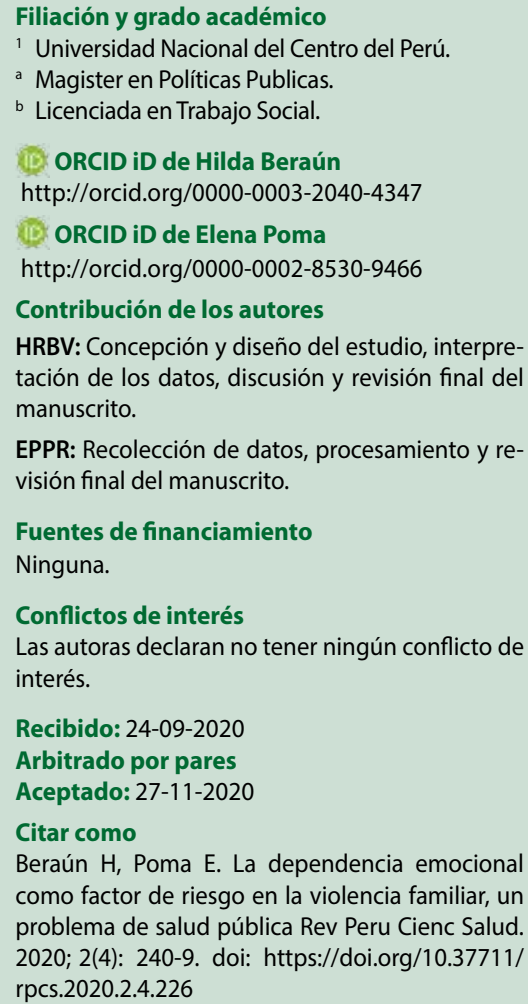

Conflictos de interés

Las autoras declaran no tener ningún conflicto de interés.

Recibido: 24-09-2020

Arbitrado por pares

Aceptado: 27-11-2020

Citar como

Beraún H, Poma E. La dependencia emocional como factor de riesgo en la violencia familiar, un problema de salud pública Rev Peru Cienc Salud. 2020; 2(4): 240-9. doi: https://doi.org/10.37711/ rpcs.2020.2.4.226

\section{RESUMEN}

Objetivo. Determinar si la dependencia emocional es un factor de riesgo para la violencia familiar de las mujeres atendidas en el Módulo Judicial Integrado en Violencia Familiar Huancayo. Métodos. Estudio cuantitativo, analítico, observacional de casos y controles. La muestra estuvo constituida por 2 grupos; el primero de 83 mujeres víctimas de violencia familiar (caso), el segundo de 21 mujeres que no reportaron violencia familiar (control). Se aplicó el cuestionario de dependencia emocional, para la prueba de hipótesis se utilizaron tablas cruzadas, a fin de analizar el odds ratio (OR). Resultados. Se determinó que el promedio de edad era de 32,5 años, el estado civil era mayoritariamente de convivientes con un $42,2 \%$, el nivel de instrucción principal, secundaria, con un $44,6 \%$, la ocupación mayoritaria, ama de casa en un $63,9 \%$ y el tipo de violencia que reportaron era física y psicológica en un $41,3 \%$ de los casos. Los niveles de dependencia en los seis factores de la escala reportaron niveles altos de dependencia emocional. según los resultados, fue de OR 13,361, IC 95 \% 4,359 - 40,950. Conclusiones. Las características sociodemográficas son un factor de riesgo para seguir en el círculo de violencia que, en un mayor porcentaje, se asocia a la agresión física y psicológica. El nivel alto de dependencia emocional explicaría los comportamientos que mantiene la mujer para retener a su pareja; una actitud que la invalida, la humilla y la mantiene sumergida en una relación violenta. Se asume que las mujeres con dependencia emocional tienen 13 veces mayor probabilidad de ser víctimas de violencia familiar que aquellas que no presentaron dependencia emocional.

Palabras clave: dependencia emocional; violencia familiar; factor de riesgo (Fuente: DeCS-BIREME)

\section{ABSTRACT}

Objective. To determine if emotional dependence is a risk factor for family violence of women treated in the Integrated Judicial Module on Family Violence of Huancayo. Methods. Quantitative, analytical, observational study of cases and controls. The sample consisted of 2 groups; the first of 83 women victims of family violence (case), the second of 21 women who did not report family violence (control). The questionnaire of emotional dependence was applied, for the test of hypothesis crossed tables were used, in order to analyze the odds ratio (OR). Results. It was determined that the average age was 32.5 years, the marital status was mostly cohabiting with $42,2 \%$, the level of primary education, secondary, with $44.6 \%$, the majority occupation, housewife in $63.9 \%$ and the type of violence they reported was physical and psychological in $41.3 \%$ of cases. The levels of dependence on the six factors of the scale reported high levels of emotional dependence; according to the results, it was OR $13.361,95 \% \mathrm{Cl} 4.359-40.950$. Conclusions. Sociodemographic characteristics are a risk factor for continuing in the cycle of violence, that, in a higher percentage, is associated with physical and psychological aggression. The high level of emotional dependence would explain the behaviors that the woman maintains in order to retain her partner; an attitude that invalidates her, humiliates her and keeps her immersed in a violent relationship. It is assumed that women with emotional dependence are 13 times more likely to be victims of family violence than those who did not present emotional dependence.

Keywords: emotional dependence; family violence; risk factor (Source: MeSH - NLM). 


\section{INTRODUCCIÓN}

La violencia es una práctica aprendida que consiste en intimidar y ejercer el control por parte de un agresor hacia su víctima. “Este modelo de poder y dominio que produce las prácticas cotidianas de violencia intrafamiliar atraviesa todas las clases sociales, niveles educativos, grupos étnicos y etarios, se da en todos los sectores de la sociedad"(1).

La violencia hacia la mujer vulnera todos sus derechos y limita el ejercicio de los Mismos. Las mujeres que las padecen se ven imposibilitadas por el abuso de poder al cual son sometidas constantemente por su pareja, las mujeres que la padecen pueden sufrir graves consecuencias en su salud. En ese sentido, "La violencia familiar es un grave problema de salud pública, debido a las consecuencias que produce en la salud física y mental de las víctimas"(2).

Presenciar situaciones de violencia familiar es un factor potencial de riesgo, en caso de los niños, para repetir esta conducta en una futura relación de pareja; en el caso de las niñas, para asumir un papel pasivo de aceptación ante la violencia sufrida ${ }^{(3)}$. La violencia de pareja es un proceso que se instaura en forma cíclica, frente al cual las víctimas que presentan vulnerabilidad psicológica desarrollan recursos de afrontamiento ineficaces, como ocultamiento, negación, resignación de la violencia o, inclusive, manifestaciones psicopatológicas como la depresión ${ }^{(4)}$.

La dependencia emocional "es la necesidad excesiva de afecto que presenta una persona hacia su pareja durante su relación, también se caracteriza por presentar comportamientos de sumisión, pensamientos obsesivos entorno a la pareja y el miedo al abandono" (5). El problema se presenta cuando estas relaciones afectivas no Funcionan y donde la ansiedad y la evitación predicen la insatisfacción en la relación de las mujeres.

Las mujeres que son sometidas a violencia presentan cuadros de ansiedad; "La ansiedad debe ser entendida como una reacción emocional ante una amenaza a nivel cognitivo, fisiológico, motor y emocional, la angustia forma una amenaza a la existencia del individuo, a sus valores morales y a su integridad física como psicológica" ${ }^{(6)}$. Esta problemática es frecuente en mujeres con dependencia emocional que se sienten incapaces de vivir solas y necesitan de una pareja para realizarse como persona en su vida cotidiana. Una de las características que presentan las mujeres con dependencia emocional es el horror y temor de quedarse solas.
La dependencia emocional explicaría la conducta de algunas mujeres víctimas de violencia de pareja, cuando justifican las infidelidades y agresiones de su pareja, cancelan procesos legales, incumplen las órdenes judiciales de alejamiento respecto a la pareja, regresan con el agresor creyendo que es posible que sus parejas tomen conciencia y cambien de actitud, y en otros casos, inician una nueva relación con características similares de malos tratos ${ }^{(7)}$.

Para atender casos de violencia familiar en la región Junín se ha implementado el Módulo Judicial Integrado en Violencia Familiar (Huancayo). Desde su implementación y funcionamiento en la ciudad, las denuncias se han incrementado en forma alarmante, ya que las víctimas que interponen la denuncia pueden acceder a medidas de protección, con una atención oportuna que brindan los juzgados de familia. Los reportes estadísticos de casos informan que, por mes, se atienden un aproximado de 120 denuncias de violencia hacia la mujer; el $50 \%$ de las cuales refieren a violencia psicológica, seguidas por un $40 \%$ de violencia física y un $10 \%$ de violencia sexual. Según reporte de valoración de riesgo, el $60 \%$ tiene riesgo severo, lo cual acontece cuando ya existen antecedentes de violencia y se ha incrementado cada vez más la agresión al punto de correr peligro su vida.

En definitiva, la intención del estudio fue apoyar a las víctimas que sufren violencia familiar y son dependientes emocionalmente de su agresor con el objeto último de contribuir a crear un programa de atención psicosocial que permita que las victimas puedan acceder a un tratamiento, mejorar su estado emocional y construir una relación armoniosa con respeto y tolerancia. Por todo lo cual, esta investigación tiene como objetivo determinar si la dependencia emocional es un factor de riesgo para la violencia hacia la mujer.

\section{MÉTODOS}

\section{Tipo y diseño del estudio}

El tipo de estudio fue cuantitativo, de nivel explicativo y de relación causal porque buscó evaluar la dependencia emocional como factor de riesgo para la violencia familiar.

El diseño fue además no experimental, analítico, observacional de casos y controles, porque buscó evaluar la relación causal entre la dependencia emocional, como factor de riesgo, y la violencia familiar. Los estudios de casos y controles parten de la presencia de un evento denominado efecto, identificando personas con la 
presencia de este y comparándolo con un grupo de personas sin la presencia del mismo ${ }^{(8)}$. Se realizó el estudio de prevalencia porque se investigó el RM, la medida de asociación o riesgo y la posibilidad de ocurrencia de un evento de interés usando probabilidades ${ }^{(9)}$.

\section{Área del estudio}

para los casos de violencia familiar, el estudio se realizó con mujeres que denunciaron ser víctimas de violencia familiar en el Módulo Judicial Integrado en Violencia Familiar de Huancayo y para los de control, mujeres de la misma ciudad.

\section{Población y muestra}

La población fue de 275 mujeres y la muestra obtenida con el procedimiento de muestreo no probabilístico intencional. Las mujeres fueron escogidas en base a criterios y juicios preestablecidos. ${ }^{\left({ }^{10)}\right.}$ El grupo de caso estuvo constituido por 83 mujeres víctimas de violencia familiar que denunciaron en el Módulo Judicial Integrado en Violencia Familiar (Huancayo), mientras que el grupo de control fue conformado por 21 mujeres del cercado de Huancayo que no presentaban violencia familiar.

\section{Procedimiento de recolección de datos}

Los datos fueron recolectados en los meses de julio, agosto y setiembre del 2019. La administración del instrumento se dio en dos grupos; uno fue de caso y el otro de control. Para las mujeres que presentaron violencia familiar (o grupo caso), el proceso fue el siguiente:

En primer lugar, se solicitó el permiso correspondiente al administrador del Módulo Judicial Integrado en Violencia Familiar (Huancayo); para aplicar la prueba a las mujeres que denunciaban ser víctimas de violencia familiar, se les explicó que se requería su colaboración voluntaria para una investigación y se les informó que a través de la encuesta se buscaba conocer si la dependendencia emocional es un factor de riesgo para la aparición de la violencia familiar. En todo caso, se aplicó a mujeres que dieron su consentimiento verbal y escrito. Para las mujeres que no presentaron violencia (grupo control), el proceso fue el siguiente:

Se les comunicó que se requería su colaboración voluntaria para una investigación porque se buscaba conocer si la dependendencia emocional es un factor de riesgo para la aparición de la violencia familiar. En todo caso se aplicó a aquellas que dieron su consentimiento verbal y escrito, también se confirmó si al momento de rellenar la encuesta deseaban o no seguir participando en la misma.

\section{Definición de variables}

\begin{tabular}{|l|c|c|}
\hline Definición operacional & Indicador & Ítems \\
\hline $\begin{array}{l}\text { Ansiedad de separación: } \\
\text { Preocupación excesiva ante la } \\
\text { posibilidad de disolución de la } \\
\text { relación. }\end{array}$ & $\begin{array}{c}\text { Temor por el } \\
\text { abandono }\end{array}$ & $\begin{array}{c}2,6,7,8, \\
13,15, \\
17\end{array}$ \\
\hline $\begin{array}{l}\text { Expresión afectiva: } \\
\text { Necesidad constante de } \\
\text { expresiones de afecto de su } \\
\text { pareja. }\end{array}$ & $\begin{array}{c}\text { Sensación de } \\
\text { inseguridad }\end{array}$ & $\begin{array}{c}5,11, \\
12,14\end{array}$ \\
\hline $\begin{array}{l}\text { Modificación de planes: } \\
\text { Dejar de hacer sus actividades } \\
\text { y estar enteramente dispuesto } \\
\text { para su pareja. }\end{array}$ & $\begin{array}{c}\text { Compartir mayor } \\
\text { tiempo con él }\end{array}$ & $\begin{array}{c}16,21, \\
22,23\end{array}$ \\
\hline $\begin{array}{l}\text { Miedo a la soledad: } \\
\text { El temor por no tener una } \\
\text { relación de pareja o sentir } \\
\text { que no es amada }\end{array}$ & $\begin{array}{c}\text { Necesidad de } \\
\text { contar con el otro }\end{array}$ & $1,18,19$ \\
\hline $\begin{array}{l}\text { Expresión límite: } \\
\text { Realiza acciones y manifiesta } \\
\text { expresiones impulsivas y de } \\
\text { autoagresión }\end{array}$ & $\begin{array}{l}\text { Llegar a hacer cosas } \\
\text { con el fin de retener } \\
\text { a su pareja }\end{array}$ & $9,10,20$, \\
\hline $\begin{array}{l}\text { Búsqueda de atención: } \\
\text { Búsqueda activa de atención para } \\
\text { asegurar la permanencia de su } \\
\text { pareja }\end{array}$ & $\begin{array}{c}\text { El centro de } \\
\text { atención en la vida } \\
\text { de su pareja }\end{array}$ & 3,4 \\
\hline
\end{tabular}

\section{Dependencia emocional}

Es un patrón persistente de necesidades emocionales insatisfechas que se intentan cubrir de manera desadaptativa con otras personas ${ }^{(11)}$.

\section{Violencia familiar}

La violencia familiar es "toda acción u omisión cometida por algún miembro de la familia en relación de poder, sin importar el espacio físico donde ocurra, que perjudique el bienestar, la integridad física y psicológica o la libertad y el derecho pleno desarrollo de otro miembro de la familia" (1).

\begin{tabular}{|l|c|c|}
\hline Definición operacional & Indicador & Ítems \\
\hline $\begin{array}{l}\text { Violencia física: } \\
\text { Es la acción o conducta, que causa } \\
\text { daño a la integridad } \\
\text { corporal o a la salud. }\end{array}$ & $\begin{array}{c}\text { Empujones, } \\
\text { golpes }\end{array}$ & 1 \\
\hline $\begin{array}{l}\text { Violencia psicológica: } \\
\text { Es la acción de control o aislamiento } \\
\text { en contra de su voluntad causa daños } \\
\text { psíquicos }\end{array}$ & $\begin{array}{c}\text { Humillaciones, } \\
\text { vejaciones }\end{array}$ & 2 \\
\hline $\begin{array}{l}\text { Violencia sexual: } \\
\text { Son acciones de naturaleza sexual que } \\
\text { se comete contra una persona }\end{array}$ & $\begin{array}{c}\text { Uso de la fuerza } \\
\text { o intimidación }\end{array}$ & 3 \\
\hline $\begin{array}{l}\text { Violencia económica: } \\
\text { La limitación de los recursos eco- } \\
\text { nómicos destinados a satisfacer sus } \\
\text { necesidades }\end{array}$ & $\begin{array}{c}\text { Privación de los } \\
\text { medios para vivir }\end{array}$ & 4 \\
\hline
\end{tabular}




\section{Instrumentos de recolección de datos}

El cuestionario utilizado fue de dependencia emocional (CDE) de Lemos y Londoño ${ }^{(11)}$. De 23 ítems, con unas alternativas de respuesta que van del 1 al 6; siendo 1. "completamente falso de mí", 2 "La mayor parte falsa de mí", 3 "Ligeramente más verdadero que falso", 4 "Moderadamente verdadero de mí", 5 "La mayor parte verdadera de mí", 6 "Me describe perfectamente". La escala mide además seis factores: ansiedad a la separación, expresión afectiva de la pareja, modificación de planes, miedo a la soledad, expresión límite y búsqueda de atención. "Los resultados arrojados a través del análisis factorial lograron identificar seis sub escalas, conformadas por ítems consistentes tanto conceptual como estadísticamente.

El análisis de confiabilidad de la consistencia interna del instrumento se realizó a través del coeficiente alpha de Cronbach que alcanzó un valor de 0,904; lo que corrobora que las puntuaciones que se obtienen en la escala de la muestra estudiada permiten tener puntajes confiables.

\section{Procedimientos}

El proceso de aplicación se realizó pidiendo su colaboración voluntaria a las mujeres que acudían al Módulo Judicial Integrado en Violencia Familiar (Huancayo). Se informó que a través de la encuesta se pretendía conocer las características demográficas, el tipo de violencia y el nivel de dependencia emocional. El otro grupo fue encuestado de manera intencional por los alrededores de la institución, se preguntó si estaban de acuerdo y a las mujeres que aceptaron, se les entregó el consentimiento informado, al mismo tiempo que a cualquier duda podían preguntar a la ejecutora. Finalmente, se agradeció por su participación en el estudio.

\section{Aspectos éticos}

Se tuvo en cuenta el consentimiento informado y la libre aceptación para ser encuestado de manera anónima, se consideró el principio de beneficencia porque la investigación ayudará a mejorar la situación de las mujeres con dependencia emocional a través de programas de atención.

\section{RESULTADOS}

Para el estudio fueron entrevistados dos grupos; el primero fue el grupo de caso mujeres que presentaba violencia familiar, mientras que el segundo grupo, de control lo conformaban mujeres que no presentaban violencia familiar. Se encontraron las siguientes características.
En la tabla 1, se observan las características demográficas de la población encuestada. El 42,2\% se encontraba en el grupo de edad de 25 a 30 años y como promedio de edad tenía 32,5. El 42,3\% manifestaba ser conviviente sin un vínculo legal con su pareja. El $44,6 \%$ de las encuestadas reportan que los estudios que alcanzaron eran de nivel secundaria, por lo que no tenían una preparación técnica para desenvolverse en el mercado ocupacional. Por último, a la pregunta sobre la ocupación que realizan el 63,9 \% respondió que era ama de casa.

En la tabla 2, se presentaron los datos demográficos. Respecto a la edad, un 52,4\% reportó tener de 31 a 36 años, con un promedio de edad de 29,6. Respecto al estado civil, el 47,6\% era soltera. Respecto al nivel de instrucción, el secundario, técnico y universitario incompleto alcanzaron el mismo porcentaje, por lo que

Tabla 1. Características demográficas (mujeres con violencia)

\begin{tabular}{|c|c|c|}
\hline Dimensiones & fi & $\%$ \\
\hline \multirow{6}{*}{$\begin{array}{l}\text { Edad } \\
\mathrm{P}(32,55) \\
\pm \mathrm{DS}(8.85)\end{array}$} & $18-24$ & 13,3 \\
\hline & $25-30$ & 42,2 \\
\hline & $31-36$ & 7,2 \\
\hline & $37-42$ & 26,5 \\
\hline & $43-48$ & 9,6 \\
\hline & $49-55$ & 1,2 \\
\hline \multirow{3}{*}{ Estado civil } & Conviviente & 42,2 \\
\hline & Casado & 38,6 \\
\hline & Divorciado & 19,3 \\
\hline \multirow{5}{*}{ Grado de instrucción } & Primaria & 18,1 \\
\hline & Secundaria & 44,6 \\
\hline & Técnica & 18,1 \\
\hline & $\begin{array}{l}\text { Superior universitario } \\
\text { incompleto }\end{array}$ & 16,9 \\
\hline & Superior universitaria & 2,4 \\
\hline \multirow{4}{*}{ Ocupación } & Ama de casa & 63,9 \\
\hline & Comerciante & 32,5 \\
\hline & Empleada pública & 3,6 \\
\hline & Total & 100,0 \\
\hline
\end{tabular}


Tabla 2. Características demográficas (mujeres sin violencia)

\begin{tabular}{lcc}
\hline Dimensiones & $\mathbf{f i}$ & $\%$ \\
\hline Edad & $18-24$ & 28,6 \\
$\mathrm{P}(29,6)$ & $25-30$ & 9,5 \\
$\pm \mathrm{DS}(6,9)$ & $31-36$ & 52,4 \\
& $37-42$ & 9,5 \\
& Soltera & 47,6 \\
Estado civil & Conviviente & 38,2 \\
& Casado & 14,2 \\
& Secundaria & 33,3 \\
Nivel de instrucción & Técnica & 33,3 \\
& Superior universitario & 33,3 \\
& incompleto & 100.0 \\
Ocupación & Estudiante & 100,0 \\
\hline & Total & \\
\hline & &
\end{tabular}

se infiere que en el $100 \%$ de los casos la ocupación es estudiante.

En la tabla 3, se observan las respuestas de las encuestadas sobre los tipos de violencia a los cuales fueron sometidas; el 41,3\% manifiesta que fue agredida física y psicológicamente, seguida de la violencia psicológica en un $19,3 \%$ de los casos y las que reportaron haber sufrido agresión física fueron el $15,4 \%$, y ya en un mínimo porcentaje del 1,9\% fueron violentadas en lo económico y sexual. Por último el $20,2 \%$ respondió no haber sufrido ningún tipo de violencia familiar.
Tabla 3. Tipos de violencia familiar

\begin{tabular}{lc}
\hline Dimensiones & $\%$ \\
\hline Violencia física & 15,4 \\
Violencia psicológica & 19,3 \\
Violencia física y psicológica & 41,3 \\
Violencia económica & 1,9 \\
Violencia sexual & 1,9 \\
Sin violencia & 20,2 \\
Total & $100 \%$ \\
\hline
\end{tabular}

En la tabla 4 se observan los resultados sobre el nivel de dependencia emocional que muestran las mujeres encuestadas. En la dimensión ansiedad a la separación, el $63,9 \%$ de los datos reportados se encuentran en nivel de dependencia alto; la dimensión expresión afectiva tiene un porcentaje del $89,2 \%$, correspondiendo a un nivel alto de dependencia; la dimensión modificación de planes, con un porcentaje de $85,5 \%$, se encuentra en un nivel de dependencia emocional alto; la dimensión miedo a la soledad, con un porcentaje de $83,1 \%$, se encuentra en un nivel alto de dependencia emocional; la dimensión expresión límite tiene un porcentaje de $77,1 \%$, por lo que se encuentra en un nivel alto, seguida por la dimensión búsqueda de atención que obtiene un porcentaje alto del $63,9 \%$. Por lo consiguiente, según los resultados las mujeres encuestadas tienen niveles altos de dependencia emocional en todas sus dimensiones.

En la tabla 5 se observan los resultados sobre el nivel de dependencia emocional que muestran las mujeres encuestadas que no presentaron violencia familiar. En

Tabla 4. Niveles de dependencia emocional (mujeres que presentaron violencia familiar)

\begin{tabular}{|c|c|c|c|c|c|c|c|c|c|c|c|c|}
\hline \multirow{2}{*}{ Nivel } & \multicolumn{2}{|c|}{$\begin{array}{l}\text { Ansiedad de } \\
\text { separación }\end{array}$} & \multicolumn{2}{|c|}{ Expresión afectiva } & \multicolumn{2}{|c|}{$\begin{array}{c}\text { Modificación de } \\
\text { planes }\end{array}$} & \multicolumn{2}{|c|}{$\begin{array}{l}\text { Miedo a la } \\
\text { soledad }\end{array}$} & \multicolumn{2}{|c|}{ Expresión limite } & \multicolumn{2}{|c|}{$\begin{array}{l}\text { Búsqueda de } \\
\text { atención }\end{array}$} \\
\hline & fi & $\%$ & fi & $\%$ & fi & $\%$ & fi & $\%$ & fi & $\%$ & fi & $\%$ \\
\hline \multicolumn{13}{|c|}{ Muy bajo } \\
\hline Bajo & 6 & 7,2 & 1 & 1,2 & & & & & 1 & 1,2 & 6 & 7,2 \\
\hline Medio & 24 & 28,9 & 8 & 9,6 & 12 & 14,5 & 14 & 16,9 & 18 & 21,7 & 24 & 28,9 \\
\hline Alto & 53 & 63,9 & 74 & 89,2 & 71 & 85,5 & 69 & 83,1 & 64 & 77,1 & 53 & 63,9 \\
\hline Total & 83 & 100 & 83 & 100 & 83 & 100 & 83 & 100 & 83 & 100 & 83 & 100 \\
\hline
\end{tabular}


Tabla 5. Niveles de dependencia emocional (mujeres que no presentaron violencia familiar)

\begin{tabular}{|c|c|c|c|c|c|c|c|c|c|c|c|c|}
\hline \multirow{2}{*}{ Nivel } & \multicolumn{2}{|c|}{ Ansiedad de separación } & \multicolumn{2}{|c|}{$\begin{array}{c}\text { Expresión } \\
\text { afectiva }\end{array}$} & \multicolumn{2}{|c|}{$\begin{array}{c}\text { Modificación } \\
\text { de planes }\end{array}$} & \multicolumn{2}{|c|}{$\begin{array}{c}\text { Miedo a la } \\
\text { soledad }\end{array}$} & \multicolumn{2}{|c|}{ Expresión límite } & \multicolumn{2}{|c|}{$\begin{array}{l}\text { Búsqueda de } \\
\text { atención }\end{array}$} \\
\hline & fi & $\%$ & fi & $\%$ & fi & $\%$ & fi & $\%$ & fi & $\%$ & fi & $\%$ \\
\hline Muy bajo & 6 & 28,6 & & & & & 2 & 9,5 & 8 & 38,1 & 9 & 42,9 \\
\hline Bajo & 10 & 47,6 & 9 & 42,9 & 13 & 61,9 & 9 & 42,9 & 10 & 47,6 & 5 & 23,8 \\
\hline Medio & 2 & 9,5 & 3 & 14,2 & 6 & 28,6 & 4 & 19,0 & 1 & 4,8 & 2 & 9,5 \\
\hline Alto & 3 & 14,3 & 9 & 42,9 & 2 & 9,5 & 6 & 28,6 & 2 & 9,5 & 5 & 23,8 \\
\hline Total & 21 & 100 & 21 & 100 & 21 & 100 & 21 & 100 & 21 & 100 & 21 & 100 \\
\hline
\end{tabular}

la dimensión ansiedad a la separación, el 47,6 \% de los datos reportados se encuentran en nivel de dependencia bajo; la dimensión expresión afectiva tiene un porcentaje del $42,9 \%$ que corresponde a un nivel bajo, mientras que el $42,9 \%$ presenta nivel alto de dependencia, la dimensión modificación de planes, con un porcentaje del $61,9 \%$, se encuentra en un nivel de dependencia emocional bajo; la dimensión miedo a la soledad, con un porcentaje del $42,9 \%$, se encuentra en un nivel bajo de dependencia emocional; la dimensión expresión límite, con un porcentaje de $47,6 \%$, que se encuentra en un nivel bajo, seguida por la dimensión búsqueda de atención $42,9 \%$, que se reporta en un nivel muy bajo. Por lo tanto, los niveles de dependencia que presentan las mujeres son niveles bajos.

En la tabla 6 se muestra que el $89,2 \%$ de los casos tienen dependencia emocional y sufren de violencia

Tabla 6. Relación de dependencia emocional con violencia familiar

\begin{tabular}{|c|c|c|c|c|c|}
\hline \multicolumn{6}{|c|}{ Tabla cruzada dependencia*violencia } \\
\hline & & & \multicolumn{2}{|c|}{ Violencia } & \multirow[b]{2}{*}{ Total } \\
\hline & & & $\begin{array}{l}\text { Con } \\
\text { violencia } \\
\text { familiar }\end{array}$ & $\begin{array}{c}\text { Sin } \\
\text { violencia } \\
\text { familiar }\end{array}$ & \\
\hline \multirow[t]{4}{*}{ Dependencia } & $\begin{array}{c}\text { Con } \\
\text { dependencia }\end{array}$ & Recuento & 74 & 8 & 82 \\
\hline & & $\begin{array}{l}\% \text { dentro de } \\
\text { violencia }\end{array}$ & $89,2 \%$ & $38,1 \%$ & $78,8 \%$ \\
\hline & $\operatorname{Sin}$ & Recuento & 9 & 13 & 22 \\
\hline & dependencia & $\begin{array}{c}\% \text { dentro de } \\
\text { violencia }\end{array}$ & $10,8 \%$ & $61,9 \%$ & 21,2 \\
\hline \multirow[t]{2}{*}{ Total } & & Recuento & 83 & 21 & 104 \\
\hline & & $\begin{array}{l}\% \text { dentro de } \\
\text { violencia }\end{array}$ & $100,0 \%$ & $100,0 \%$ & $100,0 \%$ \\
\hline
\end{tabular}

familiar; el 10,8 \% no tiene dependencia emocional pero es víctima de la violencia familiar, el 38,1 \% tiene dependencia emocional pero no es víctima de violencia familiar, con probabilidad de que en un futuro va ser víctima de violencia familiar; el $61,9 \%$, sin dependencia emocional, no presentan violencia familiar. Por lo consiguiente las mujeres que no presentan dependencia emocional, tienen menos probabilidad de ser víctima de violencia familiar.

La tabla 7 muestra los resultados obtenidos; después del análisis estadístico, el OR, entre los casos y controles, demuestra que la dependencia emocional incrementa el riesgo de padecer violencia familiar. Estadísticamente se demuestra el OR 13,361, IC $95 \% 4,359-40,950$; por lo que la probabilidad de ser víctimas de violencia familiar en mujeres que presentan niveles altos de dependencia emocional es 13 veces mayor de las que no los presentan.

Tabla 7. Estimación de riesgo de dependencia emocional y violencia familiar

\begin{tabular}{lccc}
\hline \multicolumn{3}{c}{ Estimación de riesgo } \\
\hline & Valor & \multicolumn{2}{c}{ IC del 95 \% } \\
\cline { 3 - 4 } & & Inferior & Superior \\
\hline $\begin{array}{l}\text { Razón de ventajas } \\
\text { para dependencia } \\
\text { (Con dependencia / } \\
\text { Sin dependencia) }\end{array}$ & 13,361 & 4,359 & 40,950 \\
$\begin{array}{l}\text { Para cohorte violencia } \\
=\text { con violencia } \\
\text { familiar }\end{array}$ & 2,206 & 1,328 & 3,663 \\
$\begin{array}{l}\text { Para cohorte violencia } \\
=\text { sin violencia familiar }\end{array}$ & 165 &, 078 & \\
& & & \\
N de casos válidos & 104 & & \\
\end{tabular}




\section{DISCUSIÓN}

La violencia familiar y la dependencia emocional son problemas de índole sanitaria y social porque afectan la salud mental y el desarrollo integral de las familias. En nuestra sociedad, esta problemática cada vez se agrava más por los niveles de crueldad al que son sometidas muchas mujeres. De ahí la importancia de identificar las características demográficas para realizar una intervención temprana sobre los grupos etarios más vulnerables.

En el reporte del estudio se encontró que el promedio de edad de la población fue de 29,6 años, con una distribución de 18 a 55 años. En los resultados se observa además que la edad de las mujeres víctimas de violencia familiar ha disminuido ya que se han reportado casos de violencia a partir de los 18 años. Según el INEI, 63 de cada 100 mujeres de 15 a 49 años de edad fue víctima de violencia familiar alguna vez por parte del esposo o compañero ${ }^{(12)}$. De las encuestadas, el mayor porcentaje reportó que su condición marital es conviviente. Según los estudios, las mujeres cuya condición son convivientes tuvieron más riesgo de haber sufrido violencia familiar por parte de sus parejas ${ }^{(13)}$, de lo que se deduce que el estado civil es un condicionante para que la mujer tolere maltrato de su pareja. Con respecto al grado de instrucción. Se reportó que estas alcanzaron un nivel secundario; por lo cual no cuentan con la preparación necesaria para tener un trabajo digno. Respecto a la ocupación, el mayor porcentaje reportó ser ama de casa. Esta se corrobora en otros estudios en donde concluyen que las mujeres que tienen como ocupación ser amas de casa y un nivel educativo bajo, sufren más violencia ${ }^{(14)}$. Los factores más significativos para la violencia conyugal son el psicológico y la ocupación de las víctimas ${ }^{(15)}$. Los resultados muestran que factores como la edad, estado civil, nivel de instrucción y ocupación, son igualmente determinantes para ser víctima de violencia familiar.

El tipo de violencia que reportaron sufrir las mujeres víctimas son física y psicológica en un mayor porcentaje. Este dato se corrobora en el estudio de Mulato y Poma (16), quienes hallaron que el tipo de violencia más común en las mujeres es la violencia psicológica y física. Según la teoría ecológica, los ambientes familiares cercanos son los que instigan comportamientos violentos, dado que estos pueden coadyuvar al aumento del riesgo de perpetrar y de sufrir alguna clase de violencia ${ }^{(17)}$. En la familia se da una serie de interacciones complejas $y$, si existe quiebre, esto puede desencadenar en comportamientos agresivos entre sus integrantes. La interacción de factores que contribuyen como el rechazo y el maltrato del padre, el apego inseguro a la madre $y$ la influencia de la cultura machista son determinantes para las interacciones futuras ${ }^{(18)}$.

La violencia psicológica resulta ser la más frecuente y, al mismo tiempo, la más difícil de identificar, ya que las manifestaciones suelen disfrazarse en estereotipos tradicionales sustentados por la cultura respecto a una mujer débil, que debe ser protegida por un hombre, cuyo fin último es el sometimiento. En cuanto a la violencia física, quien genera las agresiones evita dejar marcas en las partes más visibles del cuerpo, como el rostro o los brazos, procura lesionar partes en las que las probabilidades de dejar marcas sean menores o bien que estén cubiertas por la ropa, como el estómago y las piernas ${ }^{(18)}$. Pese a los intentos del Estado peruano por disminuir los casos de violencia, según el informe de la Defensoría del Pueblo ${ }^{(19)}$ se ha reportado un aumento, no solo en la cantidad de actos de violencia, sino en la crueldad y gravedad de los mismos.

Las mujeres que viven la violencia familiar tienen niveles altos de dependencia emocional. Seis son, en ese sentido los factores se encuentran más relacionados a la misma. Ansiedad de separación: se reportó un nivel alto de dependencia en este factor ya que las victimas temen la separación; por eso es que justifican sus respuestas al decir que "los insultos y el golpe es menos doloroso que la separación". Si la pareja la abandona es considerado como abrumador por la mujer dependiente y para eso realiza actividades enfocadas a retener a su pareja ${ }^{(11)}$. Esta conducta de la víctima es explicada en la teoría de vinculación afectiva de Castelló, quien refiere "que el dependiente emocional muestra una necesidad extrema de carácter afectivo, conserva lazos permanentes con el ser querido, lo cual incluye una serie de comportamientos y pensamientos obsesivos hacia la otra persona" 5 . Expresión afectiva de la pareja: el mayor porcentaje reportó tener nivel alto en el factor. Según esta dimensión, las mujeres sienten exagerada demanda de afecto y tienen altos niveles de inseguridad. Según la teoría del apego de Bowlby, la angustia es considerada como una reacción a las amenazas de pérdida y a la inseguridad en las relaciones de apego ${ }^{(20)}$.

Modificación de planes: las mujeres encuestadas reportaron tener nivel alto de dependencia en este factor, las mujeres cambian sus actividades por complacer a su pareja, tienden a alejarse de familiares y amigos, dejan de hacer sus actividades personales para hacer las actividades de su pareja, dejan su trabajo o renuncian si su pareja les solicita, acceden al aborto cuando sus parejas no desean tener al bebe, dejan de lado la atención y cuidado de sus hijos ${ }^{(3)}$. 
Miedo a la soledad; La sensación de quedarse sola: las mujeres encuestadas alcanzaron mayor porcentaje. El nivel de dependencia emocional en este factor es alto. Según Amor y Echeburúa ${ }^{(4)}$, las mujeres dependientes tienen vínculo emocional basado entre el buen y el mal trato, enamoramiento intenso, sensación de no poder vivir sin él y miedo a ser abandonada. Las personas son dependientes debido a carencias afectivas tempranas y desarrollan una vinculación afectiva intensa con personas hostiles, que no corresponden de manera satisfactoria a esos sentimientos, formándose esquemas y pautas disfuncionales sobre uno mismo y los demás ${ }^{(21)}$.

En el factor expresión límite: Se reportó un nivel alto. Las mujeres muestran dependencia extremada hacia su pareja llegando a realizar actos contra su vida con tal de mantener a la pareja a su lado. "Las mujeres con dependencia emocional amenazan a su pareja con hacerse daño al observar que su relación se siente amenazada por una posible separación; es decir son capaces de hacer cosas temerarias, llegando a arriesgar su vida con la finalidad de conservar el amor del otro"(3).

Búsqueda de atención: las mujeres reportaron tener un alto nivel de dependencia en este factor; hacen lo necesario para llamar la atención de su pareja buscan deslumbrarlo y satisfacerlo. Como lo indica Castelló ${ }^{(5)}$, las mujeres tienen necesidad excesiva de la pareja, que deriva en contactos muy frecuentes y a veces inapropiados, como llamarle frecuentemente y preguntar dónde está y qué está haciendo.

La dependencia emocional se convierte en un factor de riesgo para que la mujer tolere maltrato desde el inicio y el mantenimiento de una relación violenta por parte de su pareja. Los resultados obtenidos después del análisis estadístico el OR entre los casos y controles, demuestran que la dependencia emocional incrementa el riesgo de padecer violencia familiar. Estadísticamente se demuestra entonces, dado el OR 13,361, IC $95 \%$ 4,359 - 40,950, que la probabilidad de ser víctimas de violencia familiar en mujeres que presentan niveles altos de dependencia emocional es 13 veces mayor de las que no los presentan, de acuerdo al estudio de Manterola y Otzen ${ }^{(22)}$. Si el valor del $O R>1$, indica que existe asociación positiva; es decir, que la presencia del factor de riesgo se asocia a una mayor frecuencia de que suceda el evento. Si las posibilidades de riesgo aumentan, las victimas podrían sufrir, además un daño grave $y$, en peor de los casos, el feminicidio.

Estos datos son corroborados por González y Lea ${ }^{(3)}$, quienes demostraron que la dependencia emocional es un factor de riesgo para ser víctima de la violencia familiar. Asimismo según el MINSA (23), "el enfoque de riesgo es la probabilidad de la presencia de una o más características o factores que incrementan la aparición de la violencia". Por su parte, Espíritu ${ }^{(24)}$ Afirma que las mujeres víctimas de la violencia familiar presentan mayores niveles de dependencia emocional que las mujeres no violentadas. La probabilidad de riesgo incluye la gravedad o intensidad de los efectos que aquella produce en las victimas ${ }^{(25)}$. En la misma línea, Aiquipa ${ }^{(7)}$ concluye que existe relación estadísticamente significativa entre la dependencia emocional y la violencia de pareja.

Se concluye, indicando que los resultados obtenidos indican que algunas de las características demográficas que presentaron las mujeres víctimas de la violencia familiar: la edad promedio es de 29,6 años y los grupos de edad con mayor porcentaje fueron de 25 a 30 años; su grado de instrucción es secundaria, con poca calificación para encontrar un buen trabajo, su estado civil es conviviente, con restringidos derechos legales que le amparen como esposa; su ocupación es ama de casa, se dedican a los quehaceres del hogar completamente; el tipo de violencia que sufren es física y psicológica, con poco empoderamiento para salir del círculo de la misma.

Los niveles de dependencia emocional que presentan las mujeres víctimas de violencia familiar son altos en sus dimensiones de: ansiedad a la separación, ya que se enfocan en realizar actividades para retener a su pareja; expresión afectiva con una exagerada demanda de afecto y modificación de planes, cambian sus planes por estar con su pareja; miedo a la soledad, enamoramiento intenso, sensación de no poder vivir sin él; expresión límite, amenazan a su pareja con hacerse daño al sentirse amenazada por una posible separación; búsqueda de atención, hacen lo necesario para llamar la atención de su pareja.

Los hallazgos determinan que la dependencia emocional es un factor de riesgo para la violencia familiar. Se asume que las mujeres con dependencia emocional tienen trece veces mayor probabilidad de ser víctimas de violencia familiar que aquellas otras que no presentan dependencia emocional y tienen menos probabilidad de ser víctimas de violencia familiar. Para evitar casos de violencia familiar, se recomienda crear programas de atención oportuna de casos de dependencia emocional en mujeres. Con abordaje multidisciplinario, el servicio debe formar parte de un sistema de salud pública.

Finalmente, el estudio presentó dificultades en el tamaño de la muestra, por ser una población vulnerable con casos en procesos judiciales. 


\section{REFERENCIAS}

1. Organización Panamericana de la Salud. Informe mundial sobre la violencia y la salud: Resumen internet] [Consultado 2019 Set 10]; Disponible en :https://www. who.int/violence_injury_prevention/violence/world_ report/es/summary_es.pdf

2. Organización Mundial de la Salud. Un problema de salud global de proporciones epidémicas: Comunicado de prensa [Internet] [Consultado 2019 Set 15]Disponible en: https://www.who.int/mediacentre/news/releases/2013/violence_against_women_20130620/es/

3. González H, Lea R. Dependencia emocional como factor de riesgo para la violencia de pareja en mujeres. Distrito De San Miguelito. Panamá. Revista Tendencias en Psicología. [Internet] 2016 Dic; 1(2): 11-4 [Consultado 2019 Set 12] Disponible en: http://revistas.upagu.edu.pe/index.php/TP/article/view/332

4. Amor P, Echeburúa E, Zubizarreta I, Sarasua B. Repercusiones psicopatológicas de la violencia doméstica en la mujer en función de las circunstancias del maltrato. Revista Internacional de Psicología Clínica y de la Salud. [Internet] 2001; (2): 246-99 [Consultado 2019 Oct 10] Disponible en: https://www.redalyc.org/pdf/337/33720202.pdf

5. Castelló J. Dependencia Emocional. Características y Tratamiento. Alianza Editorial; 2005.

6. Sierra J, Ortega V, Zubeidat I. Ansiedad, angustia y estrés: tres conceptos a diferenciar. Revista Mal-estar $E$ Subjetividades [Internet] 2003; 3(1): 3-59 [Consultado 2019 Oct 11] Disponible en: https://www.redalyc.org/ pdf/271/27130102.pdf

7. Aiquipa J. Dependencia emocional en mujeres víctimas de violencia de pareja. Revista de Psicología [Internet] 2015; 33 (2): 412 -437 [Consultado 2019 Oct 11] Disponible en: http://www.scielo.org.pe/pdf/psico/v33n2/a07v33n2.pdf

8. Soto A, Cvetkvich A. Estudios de casos y controles. Rev. Fac. Med. Hum. [Internet] 2020; 20 (1): 3-10 [Consultado 2020 May 18] Disponible en: http://www.scielo.org.pe/scielo. php?pid=S2308-05312020000100138\&script=sci_arttext

9. Cerda J, Vera C, Rada G. Odds ratio: aspectos teóricos y prácticos. Rev. méd. Chile [Internet] 2013; 141(10): 2-9 [Consultado 2020 Nov 17] Disponible en: https:// scielo.conicyt.cl/scielo.php?script=sci_arttext\&pi$\mathrm{d}=$ S0034-98872013001000014

10. Arias $\mathrm{F}$. El proyecto de investigación. $6 \mathrm{a}$ ed. Episteme C.A.; 2012.

11. Lemos $M$, Londoño $N$. Construcción y validación del cuestionario de dependencia emocional en población colombiana. Acta Colombiana de Psicología [Internet] 2013; 9(2): 127-140 [Consultado 2018 Jun. 20] Disponible en: https://www.redalyc.org/pdf/798/79890212.pdf

12. Instituto Nacional de Estadística e Informática (INEI). Encuesta Demográfica y de Salud Familiar [Internet] [Consultado 2020 Set. 29] Disponible en: https://proyectos. inei.gob.pe/endes/2018/ppr/Indicadores_de_Resultados_de_los_Programas_Presupuestales_ENDES_Primer_Semestre_2018.pdf

13. Caballero J, Alfaro M, Núñez Y, Torres, H. Violencia psicológica contra la mujer por su pareja en el Perú, 2004 2007. Revista Peruana de Epidemiología [Internet] 2009; 13(3): 1-7 [Consultado 2020 Set. 20] Disponible en: https://www.redalyc.org/pdf/2031/203120367006.pdf
14. Martínez R. La violencia doméstica en la mujer de la edad mediana. Hospital Docente Ginecobstetricia de Guanabac de La Habana, Cuba. 374Rev cubana Obstet Ginecol [Internet] 2011; 37(3): 367-374 [Consultado 2020 set. 10] Disponible en: http://scielo.sld.cu/scielo.php?script=sci_arttext\&pid=S0138-600X2011000300009

15. Arce, R. Factores asociados a la violencia conyugal en el Centro Emergencia Mujer - CEM, Lima, 2017 [Internet] Lima. Universidad Nacional Mayor de San Marcos; 2019 [Consultado 2020 Set. 21] Disponible en: https://cybertesis.unmsm.edu.pe/bitstream/handle/20.500.12672/10646/Arce_cr.pdf?sequence=1\&isAllowed $=y$

16. Mulato $M$, Poma $S$. Violencia basada en género en las mujeres del Centro de Salud San Cristóbal Huancavelica 2017 [Internet] Huancavelica: Universidad Nacional De Huancavelica; 2019 [Consultado 2020 Sep. 16] Disponible en: http://repositorio.unh.edu.pe/ bitstream/ handle/UNH/2733/TESIS-2019-OBSTETRICIA-\%20MULATO\%2OSANCHEZ\%20y\%20POMA\%2OYARANGA.pdf? sequence $=1$ \&isAllowed $=y$

17. Llorens A. Cultura, familia y violencia de género: la perpetuación de la violencia contra las mujeres. [Internet] Castelló de la Plana Universidad Jaume l; 2014 [Consultado 2020 Oct. 4] Disponible en: http://repositori.uji.es/ xmlui/bitstream/handle/10234/107358/TFM_Llorens_ Aguado_antonio.pdf?sequence $=1$ \&isAllowed $=y$

18. Cuervo J, Granados M, Jiménez K. Violencia de pareja desde la teoría ecológica de Bronfenbrenner en mujeres estudiantes [Internet] Villavicencio, Meta: Universidad Cooperativa de Colombia; 2016 [Consultado 2020 Oct. 9] Disponible en: https://repository.ucc.edu.co/ bitstream/20.500.12494/12291/1/2016_violencia_pareja_teoria.pdf

19. Defensoría del Pueblo. Feminicidio en el Perú: Estudio de expedientes Judiciales. Informe [Internet] [Consultado 2020 Sep. 13] Disponible en: https://www.defensoria.gob.pe/modules/Downloads/informes/varios/2010/ informe-feminicidio.pdf

20. Ortiz E, Marrone M. La teoría del apego un enfoque actual. Revista Internacional de Psicoanálisis Aperturas [Internet] 2002; 10 [Consultado 2020 Ene 15] Disponible en: http://www.aperturas.org/articulo.php?articulo $=198$

21. Castillo E. Dependencia emocional, estrategias de afrontamiento al estrés y depresión en mujeres víctimas de violencia de pareja de la ciudad de Chiclayo. Revista Paian [Internet] 2017; 8(2):36-62 [Consultado 2020 Sep. 10] Disponible en: http://revistas.uss.edu.pe/index.php/ PAIAN/article/view/735/645

22. Manterola C, Otzen T. Valoración clínica del riesgo, interpretación y utilidad práctica. Int. J. Morphol. [Internet] 2015; 33 (14). 842-849 [Consultado 2020 Oct. 10] Disponible en: https://scielo.conicyt.cl/pdf/ijmorphol/v33n3/ art06.pdf

23. Ministerio de Salud (MINSA). Guía técnica para la atención de salud mental a mujeres en situación de violencia ocasionada por la pareja o ex pareja. Guía tecnica de atención [internet] [Consultado 2020 oct. 18] Disponible en: https://cdn.www.gob.pe/uploads/document/ file/342317/Gu\%C3\%ADa_t\%C3\%A9cnica_para_la_ atenci\%C3\%B3n_de_salud_mental_a_mujeres_en_si- 
tuaci\%C3\%B3n_de_violencia_ocasionada_por_la_pareja_o_expareja20190716-19467-n3vvt7.pdf

24. Espíritu L. Dependencia emocional en mujeres violentadas de Nuevo Chimbote. Revista de Investigación de estudiantes de psicología [Internet] 2013; 14 (18): 1-18 [Consultado 2020 Oct. 10] Disponible en: http://revistas. ucv.edu.pe/index.php/JANG/article/view/132/61
25. Puente A., Ubillos S, Echeburúa E, Paéz D. Factores de riesgo asociados a la violencia sufrida por la mujer en la pareja: una revisión de meta-análisis y estudios recientes. Anal. Psicol. [Internet] 2016; 32 (1): 295-306 [Consultado 2020 Nov 4] Disponible en: http://scielo.isciii.es/scielo.php?script=sci_arttext\&pi$\mathrm{d}=$ S0212-97282016000100034 